\title{
Effects of compositional complexity on the ion-irradiation induced swelling and hardening in Ni-containing equiatomic alloys
}

\author{
K. Jin, ${ }^{a}$ C. Lu, ${ }^{b}$ L. M. Wang, ${ }^{b}$ J. Qu, ${ }^{a}$ W. J. Weber, ${ }^{a, c}$ Y. Zhang, ${ }^{a, c}$ and H. Bei ${ }^{a *}$ \\ a Materials Science and Technology Division, Oak Ridge National Laboratory, Oak Ridge, TN \\ 37831, USA. \\ b Nuclear Engineering and Radiological Sciences, University of Michigan, Ann Arbor, MI \\ 48109, USA. \\ c Department of Materials Science and Engineering, University of Tennessee, Knoxville, TN \\ 37996, USA.
}

*Correspondence and requests for materials should be addressed to H.B. (beih@ornl.gov)

This manuscript has been authored by UT-Battelle, LLC under Contract No. DE-AC05-00OR22725 with the U.S. Department of Energy. The United States Government retains and the publisher, by accepting the article for publication, acknowledges that the United States Government retains a non-exclusive, paid-up, irrevocable, world-wide license to publish or reproduce the published form of this manuscript, or allow others to do so, for United States Government purposes. The Department of Energy will provide public access to these results of federally sponsored research in accordance with the DOE Public Access Plan.

\begin{abstract}
:
The impact of compositional complexity on the ion-irradiation induced swelling and hardening is studied in $\mathrm{Ni}$ and six $\mathrm{Ni}$-containing equiatomic alloys with face-centered cubic structure. The irradiation resistance at the temperature of $500{ }^{\circ} \mathrm{C}$ is improved by controlling the number and, especially, the type of alloying elements. Alloying with $\mathrm{Fe}$ and $\mathrm{Mn}$ has a stronger influence on swelling reduction than does alloying with $\mathrm{Co}$ and $\mathrm{Cr}$. The quinary alloy $\mathrm{NiCoFeCrMn}$, with known excellent mechanical properties, has shown 40 times higher swelling tolerance than nickel.
\end{abstract}

Keywords: irradiation, swelling, alloy, nanoindentation 
Safe, reliable, and economical operation of nuclear power plants is essential to meet rapidly increasing global energy needs. One of the key challenges from both scientific and engineering perspectives is how structural materials can retain desirable performance under extreme conditions, such as high temperature, high pressure, and high irradiation dose.[1-4] The development of fusion and Generation IV fission reactors demands materials that withstand irradiation at higher doses than do present designs—over 200 displacements per atom (dpa)—so more strict criteria are required for material selection and development.[1-3] Despite the rapid development of structural alloys used for nuclear applications over the past half-century, material degradations in harsh operating environments, e.g., severe swelling of austenitic steels, have not been fully overcome.[5, 6] Therefore, new insights into alloy design are urgently needed to resolve such challenges and problems.

Unlike traditional alloy designs, in which one major element is selected as the solvent and several dilute solute elements are added, a new family of single-phase concentrated solid solution alloys (including high-entropy alloys) has recently been developed.[7-13] These alloys, usually containing three or more principle elements equiatomically, have extreme compositional complexity, while remaining structurally simple (e.g., single-phase face-centered cubic, fcc). Their extraordinary mechanical properties have led to intensive interest in investigating their potential for practical applications, including nuclear power.[13-16] Knowledge of their irradiation response is, however, very limited, since the development of these alloys is fairly recent. 
In addition to the practical applications, these alloys open up a territory of strong scientific interest. Irradiation-induced degradations, e.g., swelling and hardening, are affected by numerous material design factors and fabrication processes, including major elements, solute additions, grain boundaries, precipitates, and cold work.[5, 17, 18]. In studies of conventional, especially commercial, alloys, these factors usually play roles simultaneously and are hardly separated. Single-phase solid solution alloys, in contrast, allow major elements in a simple crystalline form, [19] which makes them ideal model systems for studying compositional effects on the irradiation response. Furthermore, because the traditional concepts of solute and solvent are absent in these alloys, textbook alloy theories may not be suitable to describe and predict their behaviors, such as defect migration and energy transport.[14, 20]. As a result, fundamental understanding of the irradiation response of this new, attractive family of alloys is highly desired.

In this study, $\mathrm{Ni}$ and six $\mathrm{Ni}$-containing single-phase fcc equiatomic alloy single crystals- $\mathrm{NiCo}$, $\mathrm{NiFe}, \mathrm{NiCoFe}, \mathrm{NiCoCr}$, and $\mathrm{NiCoFeCr}$, as well as polycrystalline NiCoFeCrMn (with millimeter size grains) are selected. All the samples were chemically-mechanically polished by colloidal silica, achieving mirror-like surfaces with roughness below $3 \mathrm{~nm}$. In order to reach the desired radiation dose in a reasonable time scale (hours rather than years) to fulfill the urgent needs of studying these newly developed alloys,[21] instead of performing neutron irradiation, the samples were irradiated with $3 \mathrm{MeV} \mathrm{Ni}^{2+}$ ions to fluences of up to $5 \times 10^{16} \mathrm{~cm}^{-2}$. The flux was controlled at $2.8 \times 10^{12} \mathrm{~cm}^{-2} \mathrm{~s}^{-1}$, and the irradiation temperature measured at the sample surfaces was $500{ }^{\circ} \mathrm{C}$. The samples were covered by TEM grids during the ion irradiation, so that a portion 
of the sample surface was exposed to irradiation while the rest remained pristine (Figure 1a). A raster beam was used to ensure uniform irradiation, with scanning frequencies of 517 and $64 \mathrm{~Hz}$ for the horizontal and vertical direction, respectively. The dpa values were estimated using the SRIM-2013 code in the "Kinchin-Pease formalism" mode, with assuming a displacement energy of $40 \mathrm{eV}$ for all elements for convenience since the experimental values for complex alloys are currently not available. The peak dose reached $\sim 53 \mathrm{dpa}$, and the corresponding dose rate was $\sim 0.003 \mathrm{dpa} \mathrm{s}^{-1}$ (Figure $1 \mathrm{~b}$ for $\mathrm{Ni}$ as a representative).

The radiation-induced swelling was firstly studied by step-height using a Wyko ${ }^{\mathrm{TM}}$ NT9100 3D optical profilometer. The phase-shift interferometry (PSI) mode was used with sub-nm vertical resolution. An example taken from an irradiated NiCo surface is shown in Figure 1c. The microstructures (i.e. void formation) were further characterized by bright-field imaging using a JEOL 3011 transmission electron microscopy (TEM) at $300 \mathrm{keV}$. The TEM samples were prepared by the focused ion beam (FIB) lift-out method using a FEI helios Nanolab. In order to study the hardening effect under the same irradiation, a Nanoindenter XP was utilized for the nanoindentation tests, using a Berkovich triangular pyramid indenter. Tests were conducted at a constant $\dot{\mathrm{P}} / \mathrm{P}=0.05 \mathrm{~s}^{-1}$. A number of indentations were performed on each sample to allow statistical analysis, and some indents were located in the pristine region while others were in the irradiated region, as shown in Figure 1d.

Figure 2a representatively shows step-height plateaus in three dimensions for $\mathrm{Ni}, \mathrm{NiCo}, \mathrm{NiCoCr}$ and $\mathrm{NiCoFeCrMn}$ irradiated to a fluence of $5 \times 10^{16} \mathrm{~cm}^{-2}$, and the height differences are clearly 
visualized. The irradiated region of $\mathrm{Ni}$ is raised significantly above the surrounding area, indicating very large volume swelling. In contrast, the irradiated region of $\mathrm{NiCoFeCrMn}$, although it can be identified, has no observable height difference from the pristine region, indicating low volume swelling. The swelling is quantified based on the heights of the plateaus [22] acquired by line scans. Figure $2 \mathrm{~b}$ presents profiles for $\mathrm{Ni}$ at two lower fluences, 1 and $5 \times 10^{15} \mathrm{~cm}^{-2}$ in which the edges between the irradiated and pristine regions are sharp. Step-height measurements provide the total integrated swelling along the entire range modified by the irradiated ions. The overall swelling percentage is then calculated based on the step height divided by the nominal damage range $(\sim 1800 \mathrm{~nm}$, based on both simulations using the Stopping and Range of Ions in Matter [SRIM] code [23] and TEM observation). Figure 2c shows the step-height and overall swelling of $\mathrm{Ni}$ and $\mathrm{NiCo}$ as a function of ion fluence; alloying with $\mathrm{Co}$ reduces swelling by $\sim 50 \%$ at both fluences, 1.5 and $5 \times 10^{16} \mathrm{~cm}^{-2}$. The inset is a TEM image for $\mathrm{Ni}$ irradiated to a fluence of $5 \times 10^{15} \mathrm{~cm}^{-2}$, showing the void formation and the corresponding swelling that agrees to the value from the step-height measurement. The swelling curve for Ni is consistent with results for heavy ion irradiation under similar conditions. [24]

A comparison of swelling is shown in Figure $2 \mathrm{~d}$ for the highest irradiation fluence, $5 \times 10^{16} \mathrm{~cm}^{-2}$. All these fcc alloys exhibit significantly lower swelling than $\mathrm{Ni}$. Their response is unlike that of the widely used austenitic steels, e.g., Type 304 or 316, which are more susceptible to radiation-induced swelling than is $\mathrm{Ni} .[6,25]$ The NiFe binary alloy exhibits more than an order of magnitude less swelling than $\mathrm{Ni}$, suggesting that the addition of $\mathrm{Fe}$ has a stronger suppression 
effect on void nucleation and growth than the addition of $\mathrm{Co}$, similar to the behavior previously observed in $\mathrm{NiCu}$ binary alloys [26]. The two ternary alloys, NiCoFe and $\mathrm{NiCoCr}$, both have lower swelling than their mutual binary base, NiCo-; and alloying with $\mathrm{Fe}$ is more effective in reducing swelling than is alloying with $\mathrm{Cr}$. $\mathrm{NiCoCr}$ has an $\sim 20 \mathrm{~nm}$ step-height, whereas the step-height of $\mathrm{NiCoFe}$ is within the measurement uncertainty $(\sim 2 \mathrm{~nm})$ or the surface roughness. This behavior is further supported by the observation that the swelling level of $\mathrm{NiCoFeCr}$ sits between that of $\mathrm{NiCoFe}$ and $\mathrm{NiCoCr}$, suggesting competition between the $\mathrm{Fe}$ and $\mathrm{Cr}$ elements. Furthermore, the similarity between $\mathrm{NiFe}$ and $\mathrm{NiCoFeCr}$ could probably be understood as compensation between the swelling reduction due to the change from $\mathrm{Ni}$ to $\mathrm{Co}$, and the swelling enhancement due to the change from $\mathrm{Fe}$ to $\mathrm{Cr}$. Adding $\mathrm{Mn}$ into the base alloy $\mathrm{NiCoFeCr}$ - further decreases its swelling level, so that the step-height is smaller than the measurement uncertainty, similar to NiCoFe. While both sputtering effects and implanted ions cause additional uncertainties in the step-height measurements, [22] these materials were irradiated under the same conditions; therefore, the effects on these samples should be comparable.

To understand the microstructural changes that is responsible to the observed swelling, $\mathrm{Ni}$, the most swelling-sensitive material, and the alloys with the highest swelling resistance are examined by TEM. Figure 3 shows TEM images for the four materials, taken from their maximum swelling regions. Large voids, with sizes over $200 \mathrm{~nm}$, are observed in Ni but not in any of the alloys. Among the alloys, $\mathrm{NiFe}$ shows apparently larger void sizes than $\mathrm{NiCoFe}$ and $\mathrm{NiCoFeCrMn}$, while the difference between the two alloys with the highest swelling resistance is 
small (consistent with Figure 2d). Quantitatively, since the swelling increases with the cube of void diameter, the swelling values of $\mathrm{NiCoFe}$ and $\mathrm{NiCoFeCrMn}$ are lower than the others, even though their void densities are higher. The overall swelling derived from the TEM observations is also in reasonable agreement with the step-height measurements. The swelling of $\mathrm{NiCoFe}$ and $\mathrm{NiCoFeCrMn}$ are similar- -0.2 and $0.17 \%$, respectively — which is comparable to the surface roughness, or within the uncertainty of the optical profilometer in the step-height measurements. These values are about half that of $\mathrm{NiFe}(0.45 \%)$, but more than 40 times smaller than that of $\mathrm{Ni}$ $(9.4 \%)$.

The hardening of the alloys after irradiation under the same conditions is measured using nanoindentation. Differences in the load-unload curves between pristine and irradiated regions of the samples, as shown in Figure $4 \mathrm{a}$ for NiCo, indicate the change in hardness. Figure $4 \mathrm{~b}$ summarizes the measured hardness of the pristine and irradiated regions of the seven materials. The sequence of hardness of the pristine samples, from low to high, is $\mathrm{Ni}, \mathrm{NiCo}, \mathrm{NiCoFe}, \mathrm{NiFe}$, $\mathrm{NiCoFeCr}$, NiCoFeCrMn, and NiCoCr, which is similar to that of the yield strength in tensile tests. [14] The relative hardening between pristine regions and the regions irradiated to a fluence of $5 \times 10^{16} \mathrm{~cm}^{-2}$ is shown in Figure $4 \mathrm{c}$. Elemental $\mathrm{Ni}$, with the highest swelling, has the lowest hardening effect; the largest hardening is detected in NiCo, which has about half the swelling level of Ni (Figure 2d).

The difference between Ni and NiCo can be understood by comparing Figure 2c with Figure 4d, which shows the hardening of $\mathrm{Ni}$ as a function of irradiation fluence. As a result of the 
microstructure evolution under continuous irradiation at elevated temperature, the hardness of $\mathrm{Ni}$ rises with increasing fluence up to $\sim 5 \times 10^{15} \mathrm{~cm}^{-2}$, whereas it decreases when the ion fluence is higher than $1.5 \times 10^{16} \mathrm{~cm}^{-2}$, dropping to $\sim 6 \%$ hardening at a fluence of $5 \times 10^{16} \mathrm{~cm}^{-2}$. In contrast, the hardening of NiCo at a fluence of $1.5 \times 10^{16} \mathrm{~cm}^{-2}$ is close to that at $5 \times 10^{16} \mathrm{~cm}^{-2}$ (both at $\sim 35 \%$ ). Considering their swelling evolution, as shown in Figure 2c, the swelling of NiCo at the highest two fluences, 1.5 and $5 \times 10^{16} \mathrm{~cm}^{-2}$, is close to that of $\mathrm{Ni}$ for the two lower fluences, $5 \times 10^{15}$ and $1.5 \times 10^{16} \mathrm{~cm}^{-2}$, respectively; in both those cases, the hardening of $\mathrm{Ni}$ is over $20 \%$. This result indicates that the evolution of hardness in $\mathrm{Ni}$ and NiCo is similar, but the process in $\mathrm{NiCo}$ is delayed.

Such dose-dependent hardening at elevated-temperature irradiation also appeared in tensile tests. [27] The decrease in the hardening effect (although the hardness remains greater than in the pristine sample) could have several origins. For example, at high fluences, the population of dislocation loops — a strong hardening source — decreases, and thus the loop-hardening decreases. In addition, as the voids become very large at high fluence (Figure 3a), the hardness from the nanoindentation tests decreases. The trend of the Young's modulus change for Ni estimated by nanoindentation is also shown in Figure $4 \mathrm{~d}$ as a function of fluence. The decrease in the Young's modulus is due to the increased number and size of voids. [28] The hardening of the other materials lies between the values for $\mathrm{Ni}$ and $\mathrm{NiCo}$, but the values do not follow the same sequence of swelling. This finding suggests that void hardening may not be the primary hardening source under such conditions (consistent with the low swelling), and the dislocation 
(loops) may play the dominant role. For example, a significant amount of the radiation hardening in $\mathrm{NiCu}$ alloys, compared with $\mathrm{Ni}$ at similar irradiation temperatures, has been attributed to the contribution of the high density of smaller dislocation loops. [29] More detailed microstructure characterization is desired to reveal conclusively the hardening source.

Understanding the major elemental effects on swelling has been a long-standing challenge. One of the reasons is that they usually involve different perspectives of material change. For example, suppressed swelling with the addition of $\mathrm{Mn}$ has been observed in the Fe-dominant Fe-Cr-Mn-(Ni) system [18, 30]. In that case, the phase evolution and microstructure are complicated and thus factors other than the void swelling (e.g., ferrite formation and lattice parameter changes) are considered to play important roles in the density change. In contrast, the alloys investigated in this study have simple fcc structures, demonstrating that the observed material property changes result from compositional effects and associated defect properties, such as defect formation energies, migration barriers, and local and global energy landscapes. One of the most studied examples of major elemental effects on swelling is the addition of $\mathrm{Cr}$ in stainless steels (Ni-Fe-Cr systems), which has been observed to enhance void swelling. [6, 27] Increasing the $\mathrm{Cr}$ concentration [5] can decrease the effective vacancy diffusion coefficient; consequently, the supersaturation of highly mobile vacancies drives clustering, leading to smaller nucleation transient duration or incubation doses. A similar explanation has been applied to $\mathrm{Ni}$ in the Fe-Cr-Ni system [5] that increasing the Ni concentration enhances the vacancy diffusion in the Fe-dominant region, while decreasing it at the mid-Ni level. However, the mechanism of 
such elemental effects is still in debate.

In equiatomic alloys with simple fcc structure, different elements sit randomly in the crystal lattice, giving rise to extreme disturbance of local atomic, electronic, magnetic, and lattice structures. (20) Our recent studies $[20,31]$ have observed that such compositional complexity does have an impact on the irradiation response: compared with $\mathrm{Ni}$, defect evolution in $\mathrm{NiFe}$ and $\mathrm{NiCo}$ is delayed at the early stages of defect evolution at room temperature; and alloying with $\mathrm{Fe}$ can more effectively hinder defect evolution than alloying with Co. Higher-dose irradiation [31] and atomic simulation [32-34] show that the mobility of interstitial-type defects is higher in $\mathrm{Ni}$ than in the alloys, and the formation of large extended defects is suppressed in alloys, as they are not as energetically favorable as in $\mathrm{Ni}$. In the present case, the observed reduction in swelling could be related, to some extent, to the chemical disorder-induced change in vacancy diffusivity. A study of vacancy formation energy could also be interesting, as its reduction could enhance the thermal vacancy equilibrium concentration and thus greatly slow void swelling. [35]

It needs to be noted that, as a first screening test of the swelling behavior in this new family of alloys, this work identifies the need for follow-up studies to evaluate the full potential of these alloys in nuclear applications. In the present study, we only focus on one representative temperature. However, swelling is very temperature dependent, with a maximum swelling that may occur at different temperatures for different materials. [27] The possibility that the compositional complexity could change the temperature dependence of swelling requires further systematic studies of all the alloys over a wide temperature range. While step-height 
measurements have given a rapid and clear qualitative comparison on the impact of compositional complexity on the overall swelling, the TEM images have demonstrated the more quantitative effects of void size and density. More detailed cross-sectional TEM studies are needed to investigate the nature and distribution of defects (both voids and dislocation loops), as well as chemical segregation, if the mechanisms of radiation tolerance are to be revealed. Although ion irradiation has been widely used to investigate material response to radiation environments, [21] neutron irradiation experiments, while more time consuming, will ultimately be needed to conclusively confirm their behavior for nuclear applications.

Despite these issues, this study provides a new potential pathway of designing radiation-tolerant structural alloys. Traditionally, alloy design has focused on controlling the microstructures in particular alloying systems (e.g., Fe-based alloys) to achieve the desired properties, e.g., by introducing and tuning the solute atoms, precipitates, grain-sizes, and cold work. In this study, however, we demonstrate that simply modifying the compositional complexity without tuning the microstructure could also effectively improve the swelling tolerance. All the six $\mathrm{Ni}$-containing fcc equiatomic alloys have lower swelling than elemental $\mathrm{Ni}$, and their swelling is strongly affected by the compositional complexity. Here, "complexity" is used to refer not only to the number of elements but also to the types of mixing elements with electronic, magnetic, and mechanical property mismatches. $[14,20,36]$ Among the elemental variations, alloying with Co has the least swelling suppression, $\sim 50 \%$ compared with pure $\mathrm{Ni}$, whereas $\mathrm{NiFe}$ has about one order of magnitude less swelling than $\mathrm{Ni}$. NiCoFe has considerably lower swelling than $\mathrm{NiCoCr}$, 
and the value for $\mathrm{NiCoFeCr}$ lies between the two. These observations indicate that $\mathrm{Fe}$ has a stronger suppression effect than both $\mathrm{Co}$ and $\mathrm{Cr}$. The addition of $\mathrm{Mn}$ into $\mathrm{NiCoFeCr}$ further reduces its swelling, suggesting another candidate alloying element to effectively enhance swelling tolerance in Ni-based systems. Moreover, nanoindentation studies show that the hardness evolution in NiCo is delayed compared with $\mathrm{Ni}$. Our findings on controlling compositional complexity to enhance swelling resistance could act as a promising starting point, providing insights and guidance to expand the application scope of this new family of alloys as energy materials.

\section{Acknowledgements}

This work was supported as part of the Energy Dissipation to Defect Evolution (EDDE), an Energy Frontier Research Center funded by the US Department of Energy, Office of Science, Basic Energy Sciences. Ion beam work was performed at the University of Tennessee-Oak Ridge National Laboratory Ion Beam Materials Laboratory (IBML) located on the campus of the University of Tennessee-Knoxville. 


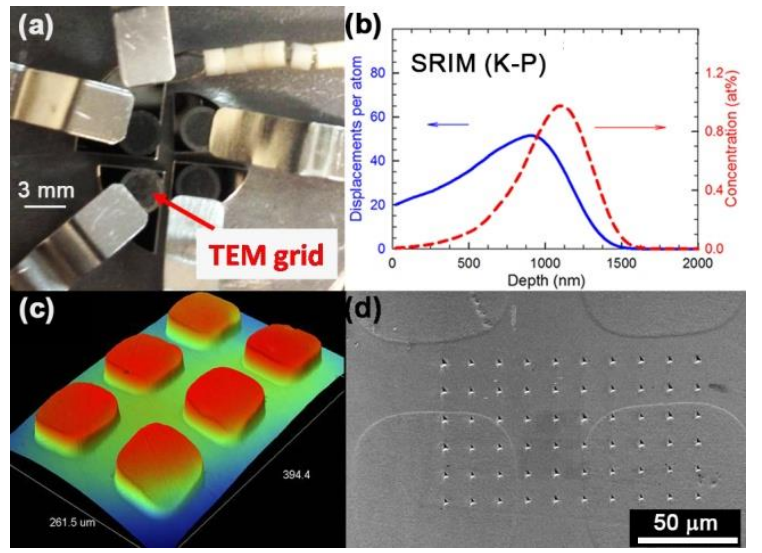

Figure 1. (a) Photo of sample arrangement for ion irradiation. (b) SRIM simulation of the displacement and implanted ion profiles for $3 \mathrm{MeV} \mathrm{Ni}$ ions in $\mathrm{Ni}$ at a fluence of $5 \times 10^{16} \mathrm{~cm}^{-2}$. (c) Surface of NiCo sample after ion irradiation, observed by an optical profilometer. (d) Indenter arrangement on the sample surface in nanoindentation tests. 

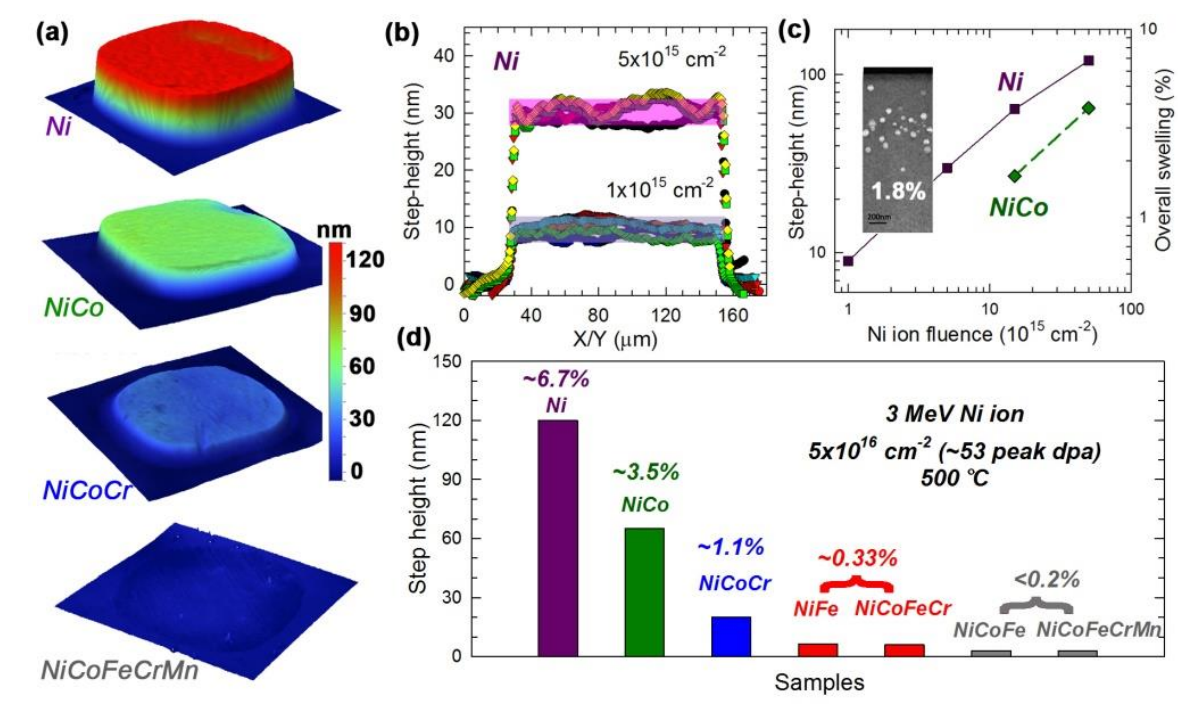

Figure 2. (a) Surface step measurements of $\mathrm{Ni}$, NiCo, NiCoCr, and NiCoFeCrMn after ion irradiation. (b) Step-height profiles for Ni under low-fluence irradiation. The spots are the data, and the bands indicate the averages and uncertainties. (c) Overall swelling from step-height as a function of ion fluence in $\mathrm{Ni}$ and $\mathrm{NiCo}$; the inset is the cross-sectional transmission electron microscopy image for $\mathrm{Ni}$ irradiated at a fluence of $5 \times 10^{15} \mathrm{~cm}^{-2}$. (d) Comparison of step-height and overall swelling among the seven materials. 


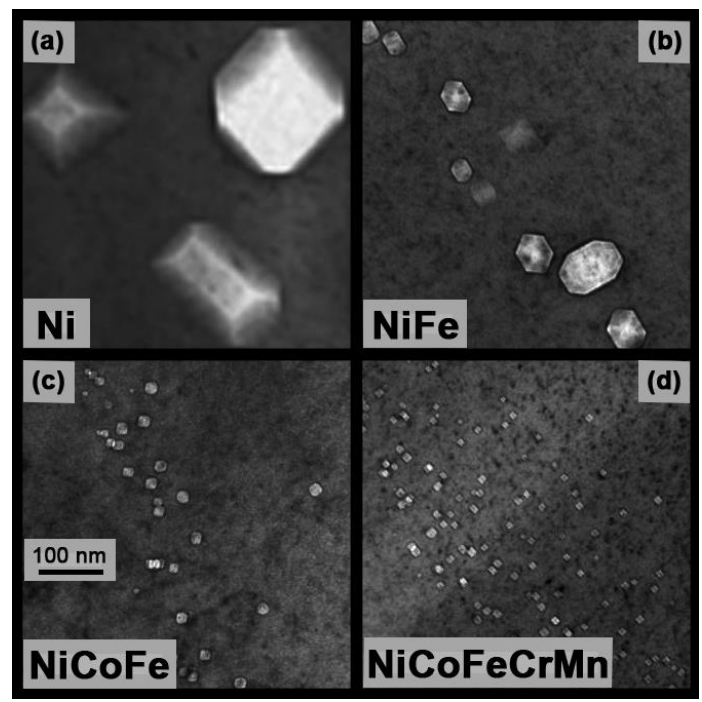

Figure 3. TEM images of the maximum swelling region of (a) $\mathrm{Ni}$, (b) $\mathrm{NiFe}$, (c) $\mathrm{NiCoFe}$, and (d) $\mathrm{NiCoFeCrMn}$ irradiated to a fluence of $5 \times 10^{16} \mathrm{~cm}^{-2}$ at $500{ }^{\circ} \mathrm{C}$. 

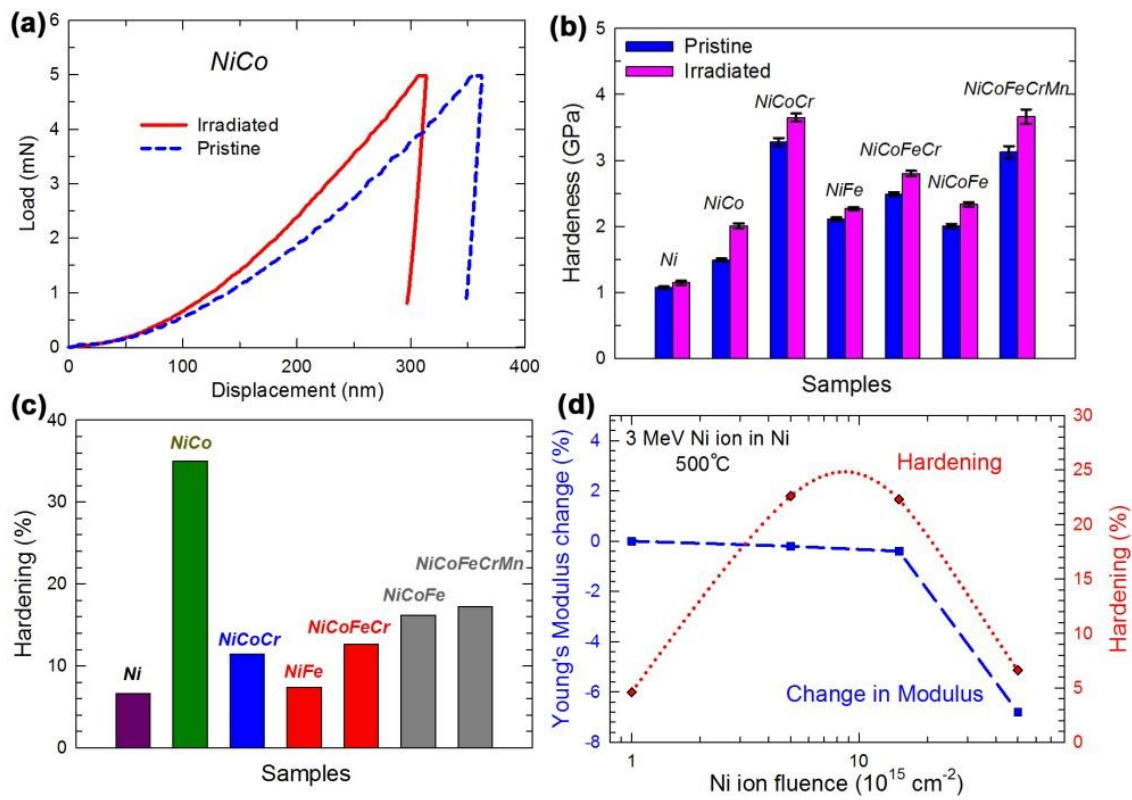

Figure 4. (a) Load-unload curves of a pristine and an irradiated NiCo. (b) Hardness before and after irradiation. (c) Irradiation-induced relative hardening. The colors used for each materials are same as in Figure 2d. (d) Change in Young's modulus and hardness of Ni as a function of irradiation fluence. 


\section{References}

[1] S.J. Zinkle, G.S. Was, Acta Mater. 61 (2013) 735-758.

[2] S.J. Zinkle, J.T. Busby, Mater. Today 12 (2009) 12-19.

[3] P. Yvon, F. Carré, J. Nucl. Mater. 385 (2009) 217-222.

[4] T. Allen, J. Busby, M. Meyer, D. Petti, Mater. Today 13 (2010) 14-23.

[5] F.A. Garner, Compreh. Nucl. Mater. 4 (2012) 33-95.

[6] F.A. Garner, M.B. Toloczko, B.H. Sencer, J. Nucl. Mater. 276 (2000) 123-142.

[7] B. Cantor, I.T.H. Chang, P. Knight, A.J.B. Vincent, Mater. Sci. Eng. A 375-377 (2004) 213-218.

[8] J.W. Yeh, S.K. Chen, S.J. Lin, J.Y. Gan, T.S. Chin, T.T. Shun, C.H. Tsau, S.Y. Chang, Adv. Eng. Mater. 6 (2004) 299-303.

[9] M.S. Lucas, G.B. Wilks, L. Mauger, J.A. Muñoz, O.N. Senkov, E. Michel, J. Horwath, S.L. Semiatin, M.B. Stone, D.L. Abernathy, E. Karapetrova, Appl. Phys. Lett. 100 (2012) 251907.

[10] O.N. Senkov, G.B. Wilks, J.M. Scott, D.B. Miracle, Intermetallics 19 (2011) 698-706.

[11] S. Guo, C.T. Liu, Prog. Nat. Sci. 21 (2011) 433-446.

[12] Y. Zhang, T. Zuo, Z. Tang, M.C. Gao, K.A. Dahmen, P.K. Liaw, Z. Lu, Prog. Mater. Sci. 61 (2014) 1-93.

[13] B. Gludovatz, A. Hohenwarter, D. Catoor, E.H. Chang, E.P. George, R.O. Ritchie, Science 345 (2014) 1153-1158.

[14] Z. Wu, H. Bei, G.M. Pharr, E.P. George, Acta Mater. 81 (2014) 428-441.

[15] S.J. Zinkle, L.L. Snead, Annu. Rev. Mat. Res. 44 (2014) 241-267.

[16] M. Caro, L. K. Beland, G. D. Samolyuk, R. E. Stoller, A. Caro, J. Alloy. Comp. (2015).

[17] L.K. Mansur, J. Nucl. Mater. 216 (1994) 97-123.

[18] F.A. Garner, H.R. Brager, D.S. Gelles, J.M. McCarthy, J. Nucl. Mater. 148 (1987) 294-301.

[19] Z. Wu, H. Bei, F. Otto, G.M. Pharr, E.P. George, Intermetallics 46 (2014) 131-140.

[20] Y. Zhang, G.M. Stocks, K. Jin, C. Lu, H. Bei, B.C. Sales, L. Wang, L.K. Beland, R.E. Stoller, G.D. Samolyuk, M. Caro, A. Caro, W.J. Weber, Nat. Commun. 6 (2015) 8736.

[21] G.S. Was, Z. Jiao, E. Getto, K. Sun, A.M. Monterrosa, S.A. Maloy, O. Anderoglu, B.H. Sencer, M. Hackett, Scripta Mater. 88 (2014) 33-36.

[22] W.G. Johnston, J.H. Rosolowski, A.M. Turkalo, T. Lauritzen, J. Nucl. Mater. 46 (1973) 273-280.

[23] J.F. Ziegler, SRIM-2013, www.srim.org.

[24] D.I.R. Norris, Rad. Effect. 14 (1972) 1-37.

[25] W.G. Johnston, J.H. Rosolowski, A.M. Turkalo, T. Lauritzen, J. Nucl. Mater. 54 (1974) 24-40.

[26] L.M. Wang, R.A. Dodd, G.L. Kulcinski, J. Nucl. Mater 155-157 (1988) 1241-1248.

[27] G.S. Was, Fundamentals of Radiation Materials Science: Metals and Alloys, Springer, Berlin, Heidelberg, New York, 2007.

[28] J.L. Straalsund, Nucl. Technol. 20 (1973) 27-34.

[29] D.H. Plantz, L.M. Wang, R.A. Dodd, G.L. Kulcinski, Metall. Trans. A 20 (1989) 2681-2687.

[30] F.A. Garner, H.R. Brager, H. Takahashi, J. Nucl. Mater. 179-181 (1991) 633-636.

[31] K. Jin, H. Bei, Y. Zhang, J. Nucl. Mater. (2015).

[32] D.S. Aidhy, C.Y. Lu, K. Jin, H.B. Bei, Y.W. Zhang, L.M. Wang, W.J. Weber, Acta Mater. 99 (2015) 69-76.

[33] L.K. Béland, G.D. Samolyuk, R.E. Stoller, J. Alloy Compond. 662 (2016) 415-420.

[34] M.W. Ullah, D.S. Aidhy, Y. Zhang, W.J. Weber, Acta Materialia 109 (2016) 17-22.

[35] C. Dimitrov, O. Dimitrov, J. Phys. F: Metal Phys. 14 (1983) 793-811. 
[36] M.C. Troparevsky, J.R. Morris, P.R.C. Kent, A.R. Lupini, G.M. Stocks, Phys. Rev. X 5 (2015) 011041. 
Graphical Abstract

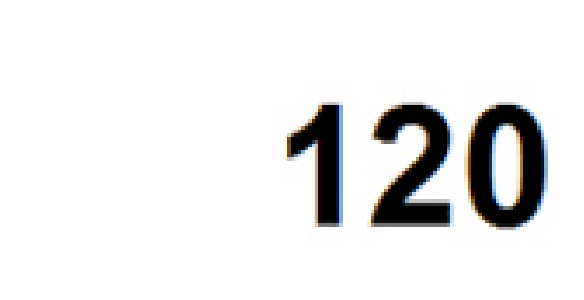

$\sim 6.7 \%$ Ni

\section{$3 \mathrm{MeV}$ Ni ion irradiation} $500^{\circ} \mathrm{C}$

$\sim 3.5 \%$ NiCo

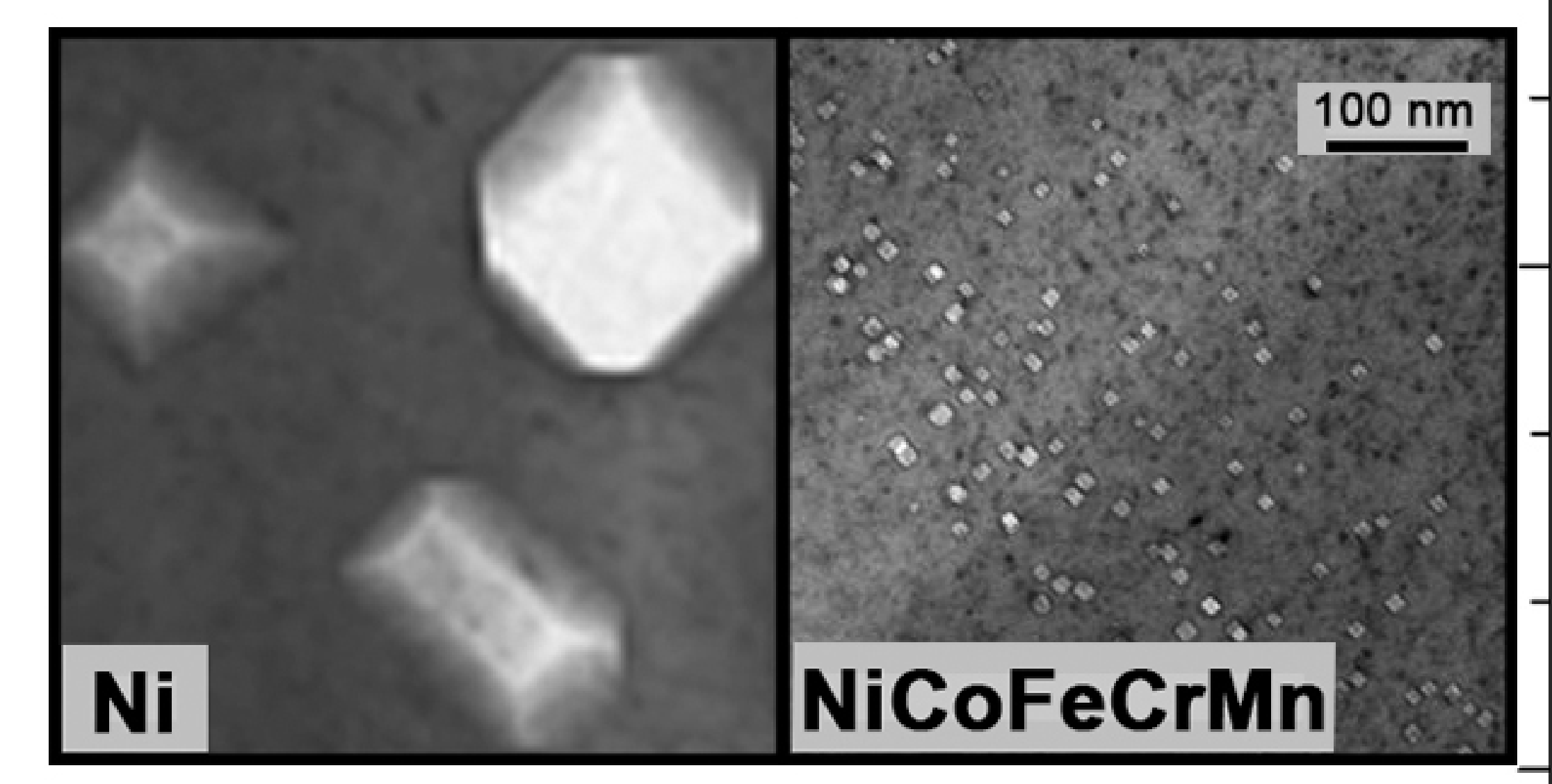

30

0 $\sim 1.1 \%$

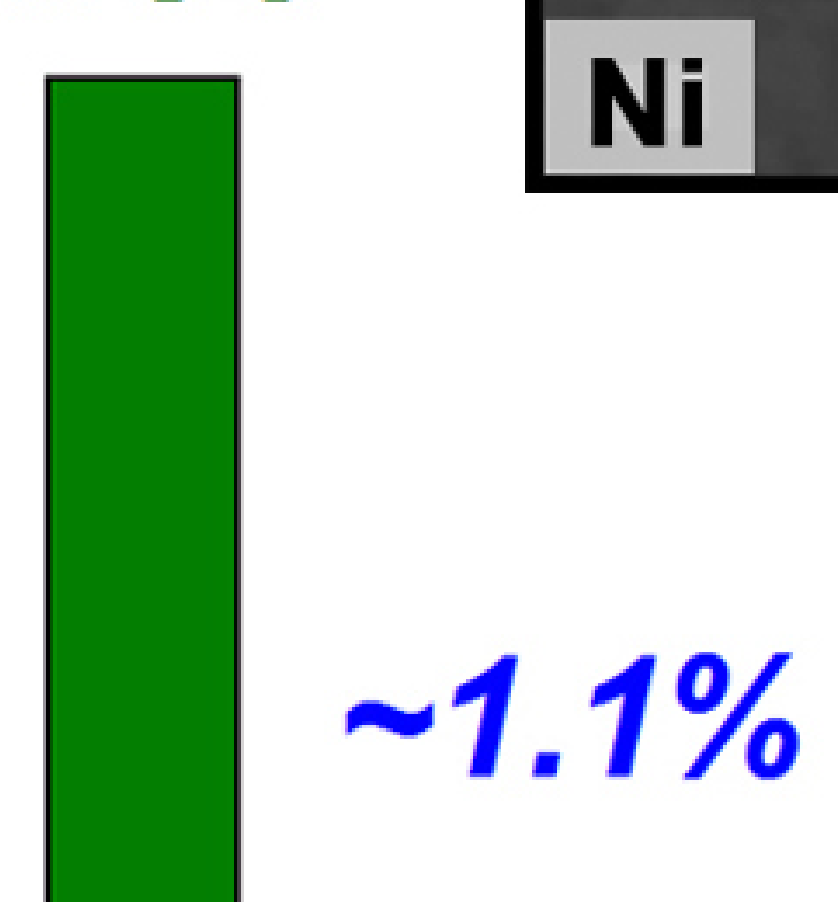

$\mathrm{NiCOCr} \quad 0.33 \% \quad<0.2 \%$ $\mathrm{NiCOFeCr} \mathrm{NiCoFeCrMn}$ $\mathrm{NiFe}$ $\mathrm{NiCoFe}$

\section{Samples}

